\title{
Fístula entero-neovejiga ileal: a propósito de dos casos
}

\author{
J.M. Pastor Guzmán, M.J. Donáte Moreno, J.M. Giménez Bachs, H. Pastor Navarro, \\ J. Lorenzo Romero, R. Ruiz Mondéjar, M. Segura Martín, A. Salinas Sánchez, J.A. Virseda Rodríguez
}

Servicio de Urologia. Hospital General Universitario de Albacete

Actas Urol Esp 2005; 29 (8): 782-786

\section{RESUMEN}

FÍSTULA ENTERO-NEOVEJIGA ILEAL: A PROPÓSITO DE DOS CASOS

Las fístulas entre el reservorio ortotópico y el tracto intestinal son de escasa incidencia (1,5-2\%). La sintomatología es variable, pero frecuentemente existe fecaluria.

Entre 90 neovejigas intestinales, presentamos dos fístulas de neovejiga a íleon, diagnosticadas en el postoperatorio próximo, mediante cistografía retrógrada; una resuelta con tratamiento conservador, y otra con cirugía abierta.

Palabras clave: Sustitución vesical. Fístula. Neocistograma. Fecaluria.

\section{ABSTRACT}

\section{ENTERO-NEOBLADDER ILEAL FISTULA: TWO CASES REPORT}

Fistulas between the orthotopic reservoir and the gastrointestinal tract have low incidence (1,5$2 \%)$. Simptomatology is variable, but it's frecuent to find fecaluria. Among 90 new intestinal bladders we show two fistule of new bladder to ileo, with nest postoperatory diagnosis, using retrograde cystografy; one was resolved with next postoperative treatment and the other with open surgery.

Keyword: Vesical substitution. Fistula. Neocystogram. Fecaluria.

$\mathrm{L}$ a ablación de la vejiga requiere indefectiblemente una derivación urinaria. La derivación urinaria al intestino ${ }^{1}$, está documentada por primera vez en 1852, cuando Simon ${ }^{2}$ realiza una ureterosigmoidostomía, en un paciente con extrofia vesical. Desde entonces se han ideado infinidad de técnicas y procedimientos con intención de evitar las complicaciones a largo plazo que provocan estas derivaciones y mejorar la calidad de vida de sus portadores.

En 1950 Bricker ${ }^{3}$ describe su técnica de ureteroileostomia, que rápidamente alcanzó una gran popularidad, fundamentalmente por su reproducibilidad, a pesar de la necesidad de bolsa colectora. Paralelamente se desarrollan y describen derivaciones urinarias continentes transintestinales, con estoma cutáneo y vaciado por autosondaje, que ya no precisa de colector externo. (Reservorio ileal de Kock, bolsa de Mainz, bolsa de Indiana, Le Bag.etc).
También a principios de la década de los cincuenta, Couvelaire ${ }^{4}$ efectúa los primeros intentos de sustitución ortotópica por intestino, pero se abandonó casi totalmente esta práctica, por los numerosos fracasos y por la gran aceptación que consiguió la ureteroileostomía. Sólo Camey y su grupo persistieron en la reconstrucción vesical funcional ortotópica, publicando sus resultados ${ }^{5}$ en 1984, siendo el punto de partida para la progresiva aceptación y mejoramiento de las sustituciones vesicales ortotópicas.

La sustitución vesical puede hacerse con distintos tramos del intestino, con intestino delgado, colon o estómago. En los últimos veinte años se ha generalizado la práctica de las sustituciones ortotópicas, ya que proporciona tan buenos o mejores resultados clínicos que las derivaciones transintestinales, continentes o no, siendo mejor aceptado por los pacientes, al evitar la servidumbre del colector. La consolidación y extensión de 
la práctica de las derivaciones intestinales, ortotópicas o cutáneas,-siendo de igual complejidad que las efectuadas hace 50 años- se debe a la depuración de la técnica quirúrgica, el advenimiento de nuevos materiales quirúrgicos, mejores conocimientos fisiopatológicos, y el gran progreso de las técnicas anestésicas.

La morbilidad en estas intervenciones, con complicaciones de todo tipo, es muy alta, entre el $30-50 \%$ de los pacientes las sufren y con reintervenciones entre endoscópicas o abiertas entre el 7-30\% $\%^{6}$. La aparición de fístulas urinarias, digestivas o mixtas se pueden producir en cualquiera de las técnicas que utilizan intestino.

Entre 90 sustituciones vesicales ortotópicas, de distinto tipo, pero con predominio de las ileales, presentamos por su rareza dos casos de fístula de neovejiga ileal a íleon terminal, uno resuelto con medidas conservadoras, y otro con cirugía abierta.

\section{CASO CLINICO}

\section{Caso 1}

Paciente varón de 70 años con antecedentes personales de diabetes en tratamiento con antidiabéticos orales, ulcus péptico, pancreatitis crónica alcohólica con varios ingresos hospitalarios por dicha patología, fumador de 30-40 cigarrillos al día desde hace 40 años pero sin criterios de EPOC.

Consultó por hematuria total intermitente, durante varios meses. Tras estudio mediante U.I.V., ecografía y TAC, se diagnosticó de Tm vesical de $3 \mathrm{~cm}$ en cara lateral izquierda. Se practicó RTU de Tm vesical y el estudio histológico demostró Ca transicional GIII, T3. Se efectuó cistoprostatectomia radical y sustitución vesical con neovejiga ileal destubulizada tipo Melchior ${ }^{7}$, con estudio histopatológico de la pieza de cistectomía de carcinoma transicional GIII, pT3 y "ca in situ"multifocal.

El postoperatorio inmediato cursa sin incidencias, con inicio de tolerancia oral al $5^{\circ}$ día; al $15^{\circ}$ día se retiran los catéteres tutores ureterales, dejando únicamente sonda vesical. Existía normal tolerancia a la alimentación oral, con tránsito intestinal y deposiciones así mismo normales. En ningún momento se detectó fecaluria ni alteraciones de las características de la orina excep- to el moco habitual en las neovejigas intestinales. A los 19 días de la intervención y siguiendo protocolo de nuestro servicio, se realizó, antes de retirar sonda, cistografía retrógrada, apareciendo paso de contraste a íleon terminal (Fig. 1).

Se diagnosticó, por tanto, de fístula de neovejiga a íleon, en paciente asintomático, con nutrición enteral bien tolerada, y sin ni siquiera alteraciones del hábito intestinal ni fecaluria.

Se optó por instaurar tratamiento conservador con dieta absoluta, nutrición parenteral, sonda nasogástrica y mantenimiento de la sonda vesical. Diez días después, -29 día postoperatorio- se realiza nueva cistografía comprobándose la estanqueidad del reservorio y la ausencia de fístula. (Fig. 2).

Tres días después se reinició la alimentación oral y se retiró la sonda vesical.

El paciente permaneció asintomático, con continencia diurna, durante tres años. Murió 40 meses después de la cistectomía por causa no bien determinada.

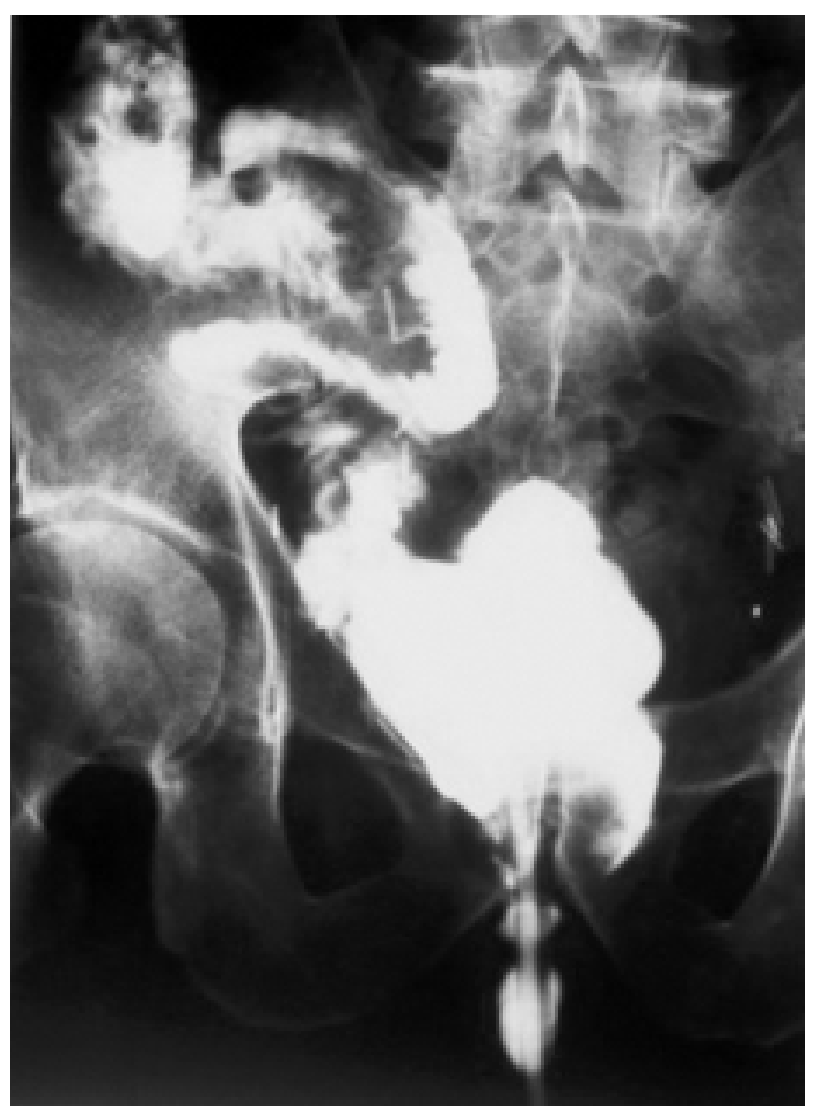

FIGURA 1. Cistografia retrógrada con fistula a ileon terminal. 


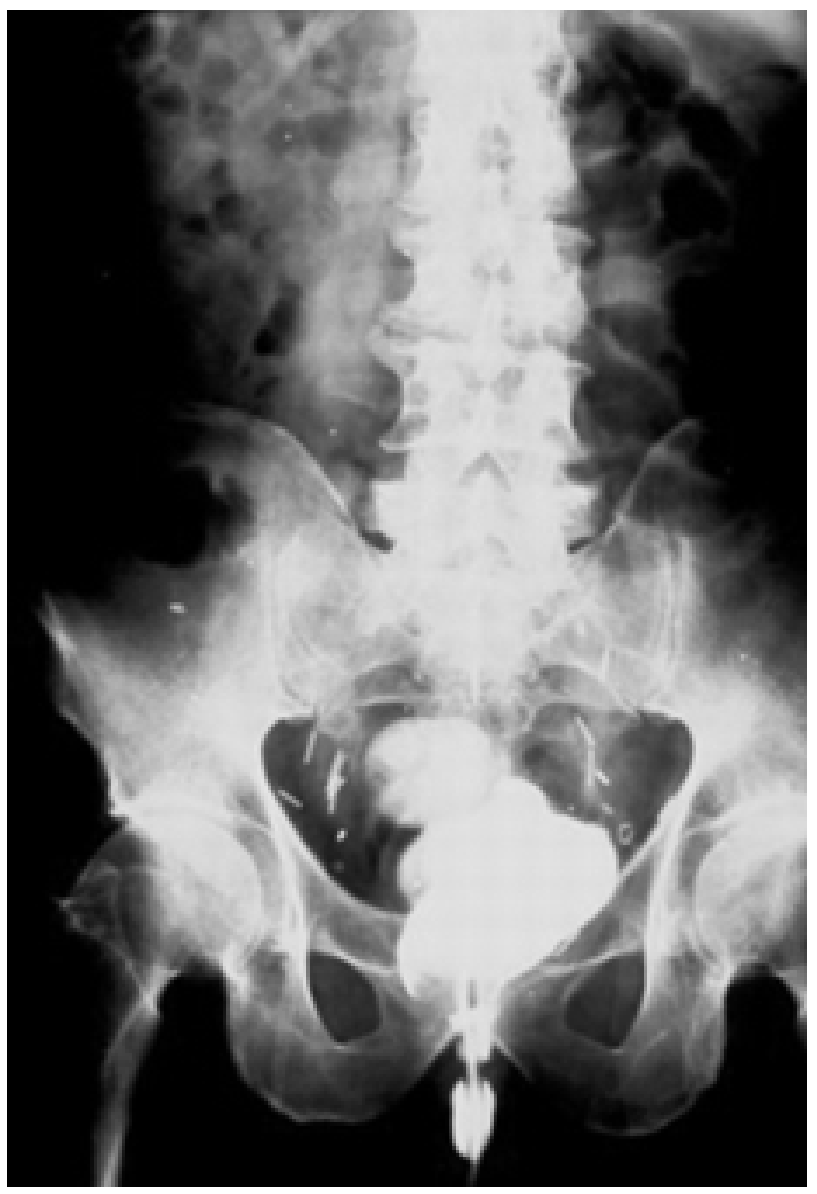

FIGURA 2. Cistografía retrógrada tras 10 dias de reposo digestivo con ausencia de fistula.

\section{Caso 2}

Varón de 66 años con antecedentes personales de: alergia a penicilinas, síndrome depresivo $\mathrm{y}$ gammapatía monoclonal de origen incierto y todavía en estudio. Por hematurias se estudia y se diagnostica de Tm vesical, siendo el resultado histológico de los fragmentos de RTU de Ca vesical infiltrante pobremente diferenciado. Se practicó Cistoprostatectomía Radical y Neovejiga Ileal con histopatología de carcinoma urotelial tipo linfoepitelióma, con bordes quirúrgicos libres. Al séptimo día de la intervención se inició alimentación oral, recuperó tránsito intestinal, pero 48 horas después comenzó con dolor abdominal, fiebre y por sonda vesical aparte del moco habitual, se apreció coloración biliosa (biliuria), que aumentaba tras la ingesta oral. En cultivos de seroma de herida, urocultivo y hemocultivo creció stafilococus aureus meticilinresistente. La cistografía retrógrada demostró la fístula de neovejiga a íleon. (Fig. 3).

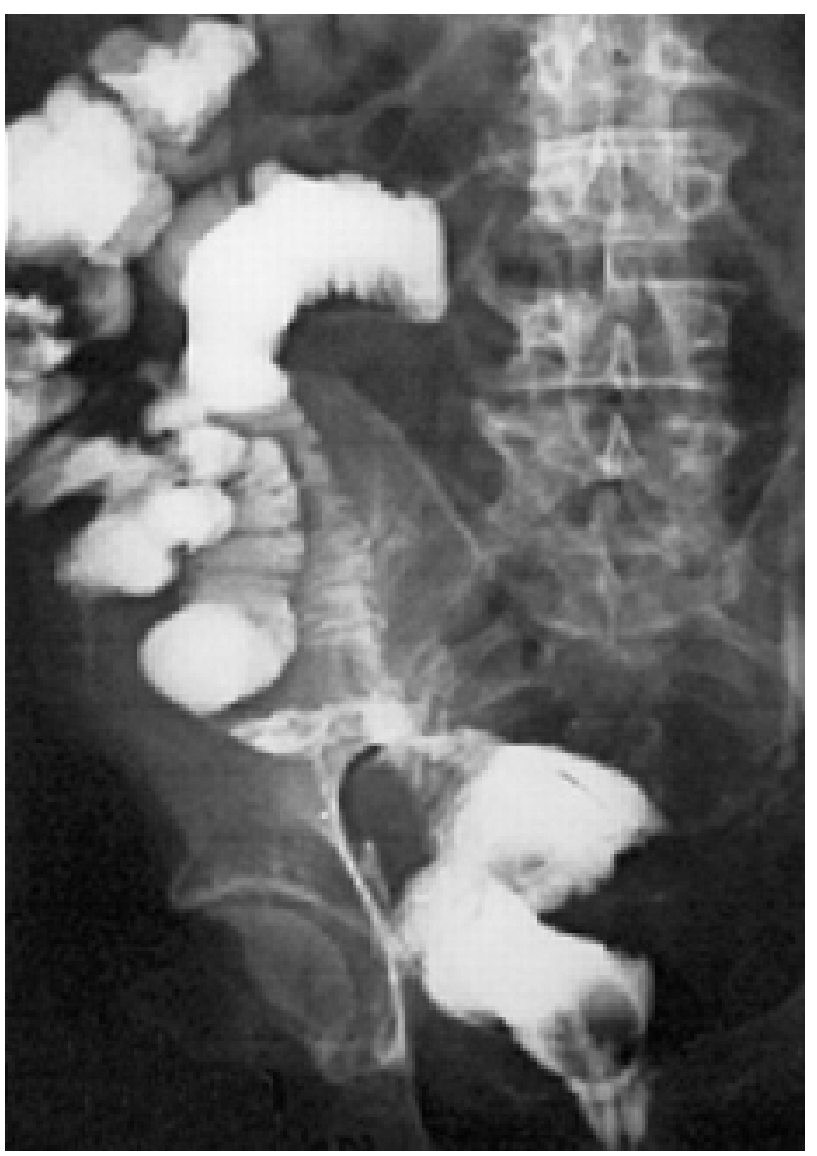

FIGURA 3.Cistografia retrógrada ( $2^{\circ}$ caso) con fistula a ileon terminal.

El tratamiento inicial fue conservador, con sonda vesical, reposo digestivo y nutrición parenteral, persistiendo la fístula en dos cistogramas posteriores, el último 7 semanas después del primero. Por ello se decidió intervención quirúrgica, procediéndose resección ileal y anastomosis terminoterminal y cierre del cuerno de neovejiga fistulizado. En cistografía de control 12 días después (Fig. 4), se confirma la desaparición de la fístula y fue alta hospitalaria con buen estado general a los 70 días del ingreso.

Cinco meses después, ingresa de urgencia con fracaso renal agudo, deterioro brusco del nivel de conciencia y crisis comiciales complejas (status epiléptico?), que precisó de intubación, ventilación mecánica y diálisis, comprobando por ecografía ectasia renal bilateral, colocándose nefrostomía percutánea bilateral. Mejoró la función renal y recuperó estado de conciencia. Posteriormente se intentó cateterismo ureteral retrogrado y anterógrado que resultó infructuoso, por 


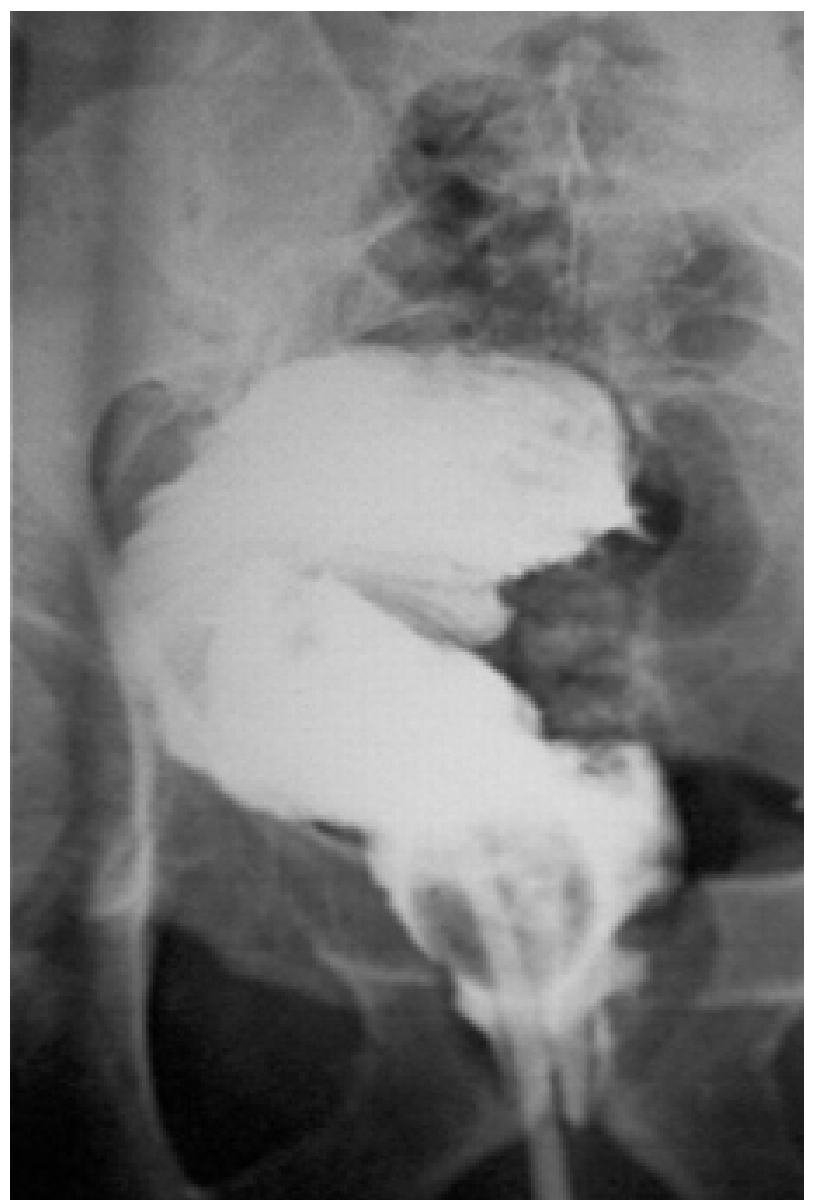

FIGURA 4. Cistografia retrógrada ( $2^{\circ}$ caso) 12 dias después de reparación quirúrgica con ausencia de fistula.

estenosis a nivel de anastomosis ureterointestinales. Ya no precisa de diálisis con niveles de creatinína entre 1,4-1,8.

Actualmente el paciente 2 años después de la cistectomía, aunque con buen estado general y sin progresión de su enfermedad, es portador de sonda vesical y de nefrostomía bilateral, no aceptando por el momento ninguna alternativa quirúrgica tendente a liberarle de los catéteres.

\section{DISCUSION}

La aparición de fistulas digestivas, urinarias o mixtas, es posible en las derivaciones urinarias con utilización de intestino. En las fistulas enterourinarias la presentación clínica más común es la fecaluria $^{8}$. Pueden ser precoces, (hasta 3 meses tras la cirugía) o tardías. La etiología de la fistula parece ser multifactorial $^{8}$, siendo la radioterapia preoperatoria $^{8,9}$, el factor de riesgo principal, pero otras causas predisponentes son la diabetes, reservorio mal vas- cularizado, anastomosis a tensión, deficiente estado nutricional, presencia de tumor o cuerpos extraños y sobredistensión del reservorio especialmente por la obstrucción de la sonda por moco, en el postoperatorio próximo, o la retención crónica en el tardío. $\mathrm{El}$ diagnostico ante la sospecha clínica se hace con el neocistograma; sólo se realizará TAC si se sospecha obstrucción intestinal o absceso, y en las tardías para descartar recurrencias. El tratamiento inicial debe ser conservador, si no existen signos de sepsis o abdomen agudo que obliguen a exploración quirúrgica urgente ${ }^{10}$, el más empleado es el reposo digestivo, la nutrición parenteral y el drenaje permanente prolongado de la neovejiga. El tiempo necesario para el cierre de la fístula no está determinado, no obstante, parece indicado el tratamiento quirúrgico si en el reservoriograma persiste la fístula después de 8-12 semanas.

Las fístulas conducto o reservorio entéricas son poco frecuentes, entre el $1.5-2 \%^{11-13}$, pero se dan en todo tipo de derivaciones, desde la ureteroileostomía cutanea ${ }^{14}$, reservorios continentes cutaneos $^{8,11}$, hasta las sustituciones ortotópi$\operatorname{cas}^{12,13,15,16,17}$

En el primero de nuestros casos, no se produjo fecaluria y el paciente tuvo un postoperatorio sin incidencias descubriéndose la fístula al hacer el reservoriograma previo a la retirada de la sonda vesical; el tratamiento fue conservador y con resolución de la fístula en breve tiempo. El segundo caso que presentamos debutó con fecaluria-biliuria a los pocos días de la cistectomía, no se resolvió con medidas conservadoras y requirió de reintervención. Ninguno de los dos pacientes recibió radioterapia preoperatoria.

\section{REFERENCIAS}

1. Hendren Wh. Perspectiva histórica de la utilización del intestino en urología. Clínicas de Urología de Norteamérica. 1997;4:803-814.

2. Simon J. Ectopia vesicae (Absence of the anterior walls of the bladder and pubic abdominal parietes); operation for directing the orificies of the ureter into the rectum; temporary success; subsequent death; auptopsy. Lancet, $1852 ; 2: 568$

3. Bricker Em. Bladder substitution after pelvic evisceration.Surg Clin North Am 1950;30:1511.

4. Couvelaire R. Le reservoire ileal de substitution aprés cystectomie totale chez l'homme. J Urol et Nephrol, 1951;57:408-417.

5. Lilien OM, Camey M.25 year experience with replacement of the human bladder. J Urol 1984; 132:886. 
6. Montesino Semper M, Santiago González de Garibay A, Ruiz Ramo M, Hualde Alfaro A, Jiménez Calvo J, de Pablo Cárdenas A, Pinós Paul M, Lozano Uruñuela F. Sustitución vesical ortotópica tipo Camey II y sus complicaciones: 10 años de experiencia. Actas Urol Esp2001;25(2):99-104.

7. Melchior H, Spehr Ch, Knop-Wagemann I, Person Mch, Juenemann Kp. The continent ileal bladder for urinary tract reconstruction after cystectomy. A survery of 44 patients. J Urol 1988:139:714

8. Ubirajara Barroso Jr, Da Silva EA, Alvin Barroso V, Gomes De Olive, Edson Pontes J. Diagnostico y tratamiento de la fístula enteroneovesical postcistectomía. Actas Urol Esp 1999;23(7):573-578

9. Camey M,Botto H,Richard E.Complications of the Camey procedure.Urologic Clinics of North America1988;15(2): 249-255.

10. Kevin P Killen, John A Libertino.Management of Bowel and Urinary Tract Complications After Urinary Diversion. Urologic Clinics of North America 15(2)1988:183-194.

11. Tarter Th, Kawachi Mh, Wilson Tg.Enteropouch fistula: a rare complication of right colon continent urinary diversion. J Urol 1995; 154:364-366

12. Hautmann Re, De Petricone R, Gottfried Hw, Kleinschmidt K, Matt Paiss. The ileal neobladder: complications and functional results in 363 patients after 11 years of followup. $J$ Urol 1999;161(2):422-428.
13. Flohr P, Hefty R, Paiss T, Hautmann R.The ileal neobladderupdated experience with 306 patients. World J Urol 1996; 14:22-26.

14. Mc Callion WA Wilson BG, Kennedy JA, Keane Pf. Ileal conduct.enteric fistula.Br J Urol 1993;71:230-231

15. Deleveliotis Ch, Picramenos D, Macriochoritis C, Kiriazis P, Kostakopoulos A.Ilioneobladder-enteric fistula: a rare early postoperative complication treated conservatively. $\mathrm{Br}$ J Urol 1995;76:407-408.

16. Ng Cs, Klein A. Conservative management of an ileal neobladder-enteric fistula. Urology 1999;54(2):366-

17. De Pablo Cárdenas A, Jiménez Aristu JI, Pinós Paul MA, Jiménez Calvo JM, Lozano Uruñuela F, Villanueva Pérez JI, Santiago González se Garibay AM. Fístula enteroneovesical. Imágenes en urología. Actas Urol Esp 2004;28(4): 333.

Dr. J.M. Pastor Guzmán

San Antonio, 47 - 4ํㅡㄹ Dcha.

02001 Albacete

E-mail: jmpastorg@hotmail.com

(Trabajo recibido el 14 de diciembre 2004) 